\title{
Autonomous Drone System Development for Power Transmission Line Inspections
}

\author{
Nobuhiro Kishigaki, a, ${ }^{a}$, Norihiko Tsutsumi ${ }^{a}$, Toshikazu Teshigawara ${ }^{a}$, Daisuke Ota ${ }^{a}$, Hiroyuki Miyoshi ${ }^{b}$, \\ Kensei Yamamoto ${ }^{\mathrm{b}}$, Fumito Minoura ${ }^{\mathrm{b}}$, Tomonori Shiraishi ${ }^{\mathrm{b}}$, Tsuyoshi Chiba ${ }^{\mathrm{c}}$, Masayuki Kumadac, \\ Wiktor Piwowarczyk ${ }^{\mathrm{c}}$, Takayuki Dewa ${ }^{\mathrm{d}}$
}

${ }^{a}$ R\&D Dept. TEPCO Research Institute, Tokyo Electric Power Company Holdings, Inc. 4-1, Egasaki-cho, Tsurumi-ku, Yokohama, 230-8510, Japan

${ }^{\mathrm{b}} \mathrm{TEPCO}$ Power Grid, Inc.

1-1-3, Uchisaiwai-cho, Chiyoda-ku, Tokyo 100-8560, Japan

'Blue innovation Co., Ltd.

4F, Ichigo-Hongo Bldg., 5-33-10, Hongo, Bunkyo-ku, Tokyo 113-0033, Japan

dTEPCO SYSTEMS CORPORATION.

2-37-28, Eitai, Koto-ku, Tokyo 135-0034, Japan

*Corresponding Author: kishigaki.nobuhiro@tepco.co.jp

\begin{abstract}
Conductors and earth wires of overhead power transmission lines are visually inspected from the ground using high-magnification scopes, etc., but it takes time, labor, and a high degree of concentration not to overlook faults over the entire length of the lines.

With the aim of resolving this issue for more efficient inspections, we have developed an autonomous drone system for power transmission line inspections, in which a drone autonomously flies along the sag of power lines and captures images of them to check for faults.

This time, we captured images of actual charged power lines using the autonomous drone system developed for power transmission line inspections. This paper reports our results.
\end{abstract}

Keywords: Drones, overhead power transmission lines, power line inspection.

\section{Introduction}

Conductors and earth wires of overhead power transmission lines are exposed to various natural environments, and are likely to suffer disconnection of element wires, melting, corrosion, and other faults, as shown in Fig. $1^{(1)}$. To check for these flaws, visual inspections are performed from the ground using high-magnification scopes, etc., but it takes time, labor, and a high degree of concentration so as not to overlook faults over the whole length of the power lines.

To cope with this issue, we have been working on the development of an autonomous drone flight technology with the aim of using drones (UAV: Unmanned Aerial Vehicles)-which have recently been utilized in a variety of industrial fields-in the inspection of conductors and earth wires of overhead power transmission lines. This system is especially for the inspection of single-conductor transmission lines, and a drone flies along the sag of power lines and captures images of them while ensuring a fixed separation distance from the lines ${ }^{(2,3)}$.

Since conductors and earth wires of overhead power transmission lines sag down, it is necessary to fly a drone along the wires to be photographed while ensuring a fixed distance and angle between the wires and the drone so that zoom magnification and the focal length of the camera do not deviate from the objects. However, manually controlling a drone along power lines requires very advanced technique and takes time. It is also difficult to set waypoints along the power lines in advance, because the sag of the conductors of overhead power transmission lines changes depending on the outside air temperature and the amount of current.

To overcome this issue, we developed an autonomous 
drone system for power transmission line inspections, in which a drone autonomously flies along the sag of power lines while ensuring an optimum clearance from the lines by detecting them using a millimeter-wave radar, and captures images of the power lines. We captured images of power lines with the autonomous drone system for power transmission line inspections while ensuring such clearance, which previous research ${ }^{(4,5,6)}$ had examined using an uncharged electric power transmission system for training and obtained good results ${ }^{(7)}$.

As shown in Fig. 2, the system used for the test is a single-conductor transmission line with a voltage class of $66 \mathrm{kV}$, a span length of $60 \mathrm{~m}$, and a conductor type of ACSR $240 \mathrm{~mm}^{2}$ (outer diameter $22.4 \mathrm{~mm}$ ), and there was no difference in the height of supporting points of conductors. On the other hand, in the actual commercial lines in service, the length of spans is longer and there are points where there is a large difference in height of supporting points. In this experiment, we captured images of actual charged power transmission lines using the newly developed autonomous drone system for power transmission line inspections and verified the applicability of the system to the inspection of conductors and earth wires.

\begin{tabular}{|c|c|c|c|}
\hline Fault type & $\begin{array}{c}\text { Disconnection of } \\
\text { element wire }\end{array}$ & Molten part & Corrosion \\
\hline $\begin{array}{c}\text { Outer } \\
\text { appearance }\end{array}$ & & & \\
\hline
\end{tabular}

Fig. 1: Power line faults

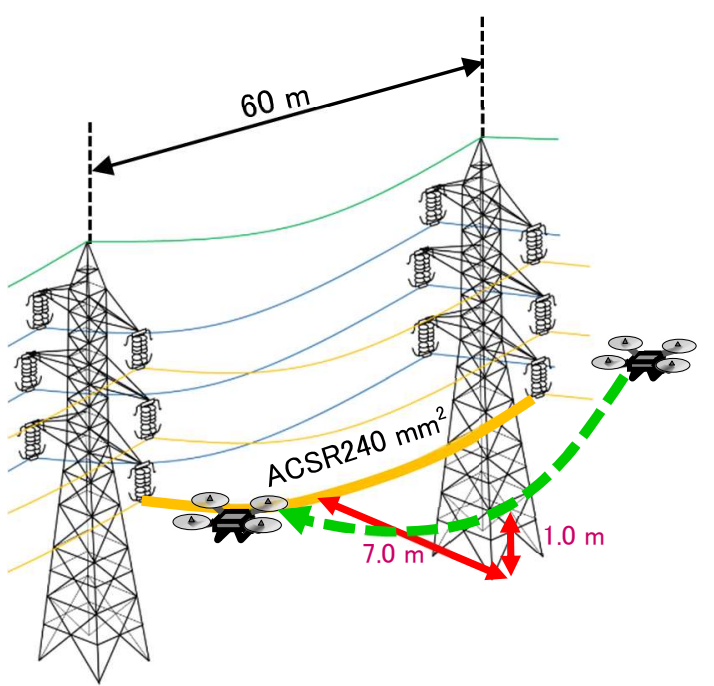

Fig. 2: Overview of flight along power lines and photography test

\section{Methods}

\subsection{Overview of Autonomous Drone System for Power Transmission Line Inspections}

A tablet device and a proportional controller are used to operate the developed autonomous drone system.

The operating device of the autonomous drone system for power transmission line inspections, which is shown in Fig. 3, is used to input flight routes in advance, to activate the autonomous system, to enter commands to start and finish flights along power lines, and other operations necessary for photographing power lines.

On the other hand, the proportional controller is used in the event of an emergency to switch the operation mode from automatic control to manual and safely bring the drone back to and land it at the takeoff point.

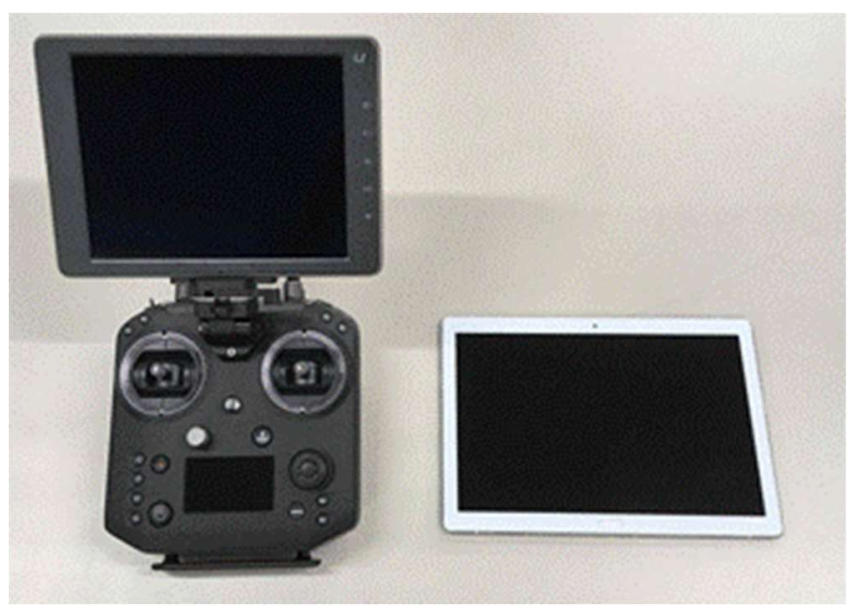

Fig. 3: The proportional controller and operating device of the autonomous drone system for power transmission line inspections.

\subsection{Drone for Power Transmission Line Inspections}

To fly a drone along the sag of power lines while keeping a certain distance and angle from the lines, it is necessary to grasp the position of the lines in real time. To achieve this, we use a millimeter-wave radar, which is lighter than other sensors, consumes less power, and is not affected by the background of the object. Fig. 4 shows the millimeter-wave radar mounted on the drone.

The drone used is the DJI Matrice 210 RTK (Fig. 5). A millimeter-wave radar is mounted on the upper part of the drone. To control the flight of the drone, a microcomputer calculates the traveling direction of the drone unit based on the position information of the power lines detected by the millimeter-wave radar mounted on the drone, and transmits 
it to the flight controller using Onboard SDK.

The camera and gimbal used are both DJI Zenmuse 30 (Fig. 6). The gimbal is controlled so as to face the direction of the conductors and earth wires to be photographed based on the position information of the power lines detected by the millimeter-wave radar on the drone.

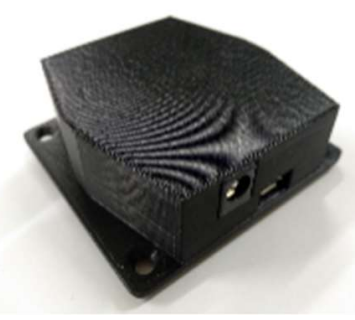

Fig. 4: External appearance of the millimeter-wave radar

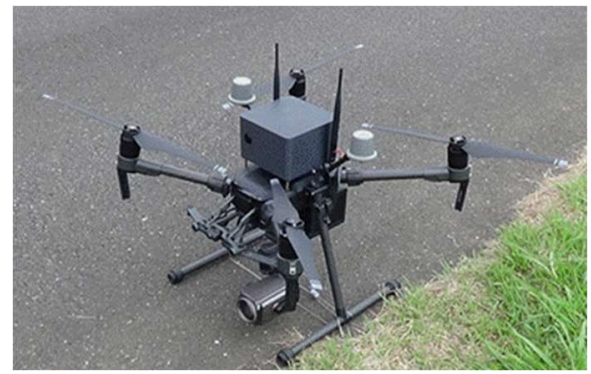

Fig. 5: External appearance of the drone

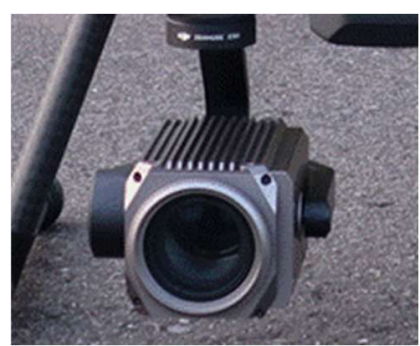

Fig. 6: External appearance of the gimbal camera

\subsection{Flight Verification}

Flight verification was carried out with commercial lines that are actually in operation. The following two systems were used in the flight verification.

- Verification Location 1: HDCC $150 \mathrm{~mm}^{2}$ single conductor (external diameter: $16 \mathrm{~mm}$ ), Tmax $=24100 \mathrm{~N}$, span length $=187 \mathrm{~m}$, difference in height of support points $=26.6 \mathrm{~m}$ (Fig. 7)

- Verification Location 2: HDCC $150 \mathrm{~mm}^{2}$ single conductor (external diameter: $16 \mathrm{~mm}$ ), Tmax $=24100 \mathrm{~N}$, span length $=365 \mathrm{~m}$, difference in height of support points $=7.0 \mathrm{~m}$ (Fig. 8)
Flight verification was conducted for 6 days from October to November 2020. The total flight time was 240 minutes. The weather was mild during the verification period, and the maximum wind speed was about $5 \mathrm{~m} / \mathrm{s}$ on the ground surface.

In the flight verification, the drone detected a power line to be photographed near a steel tower and was flown $1.0 \mathrm{~m}$ above the $7.0 \mathrm{~m}$ side from the power line while maintaining a certain level of clearance from the line. It automatically flew along the sag of the power line at a speed of $0.7 \mathrm{~m} / \mathrm{s}$, and captured images of it.

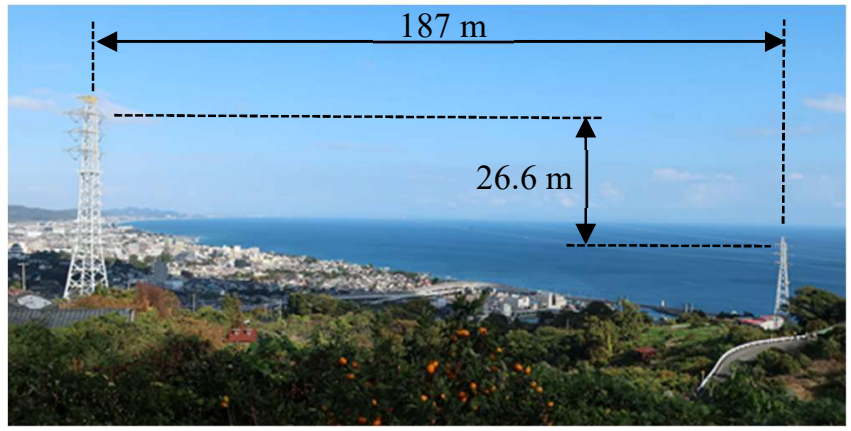

Fig. 7: Verification Location 1

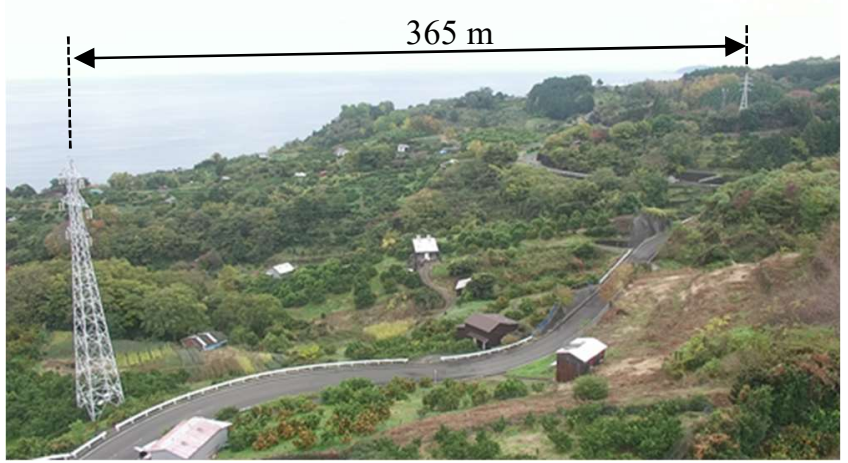

Fig. 8: Verification Location 2

\section{Results and Discussion}

\subsection{Results of Flight Verification}

In the early stages of the flight verification at both of Verification Locations 1 and 2, the electric wire sometimes came off from the angle of view of the camera. However, as a result of repeated flight and adjustment, it improved.

Overall, all of the power lines, from the start point to the end point of the flight along power lines, were in the angle of view of the camera and recorded, and the results were good. Fig. 9 shows an image of a power line taken from a flying drone with a $7 \mathrm{x}$ zoom. The element wires can 
be recognized in the image of the power line, and it can be used for actual power line inspections.

In addition, the maximum wind speed of $5 \mathrm{~m} / \mathrm{s}$ during the verification period made autonomous flight impossible, and there was no need to switch to manual control.

In this study, a millimeter wave radar was mounted on the drone to detect power lines. The millimeter wave radar is distinctive for being lightweight, low in power consumption, comparatively low cost, and not affected by backlight.

In some previous research ${ }^{(8)}$, LiDAR was used as the sensor for the detection of power lines, but it is comparatively heavy and very expensive. Furthermore, it might not be able to detect thin objects like power lines from a distance. This may be an obstacle to the application of the system to the inspection of extra-high voltage transmission lines where a larger clearance should be ensured.

There was also a previous study ${ }^{(9)}$ in which power lines were detected by analyzing recorded images, and the gimbal was controlled so that the target power lines would not deviate from the angle of view of the camera to fly a drone along power lines. However, there is the risk that power line detection errors may occur due to backlight, or depending on the background, that the sensor may erroneously detect power lines.

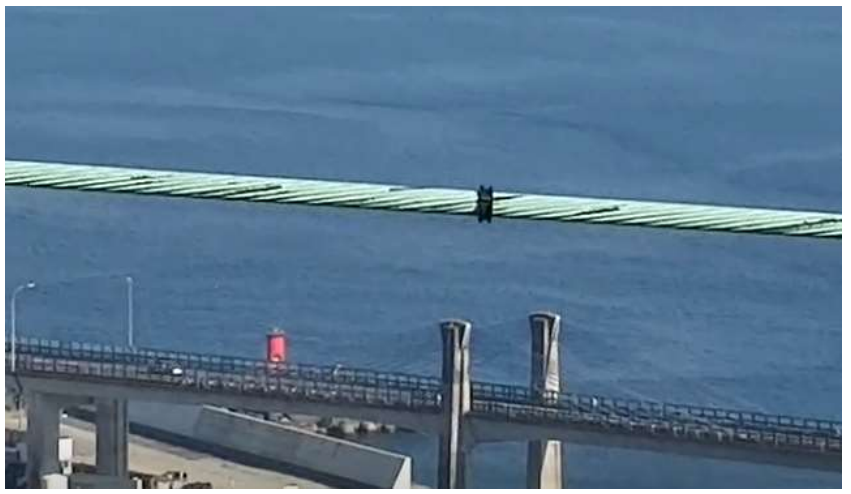

Fig. 9: Power line image ( $7.0 \mathrm{~m}$ from the electric wire)

\subsection{Future Challenges}

\section{(a) Drone weight}

The drone unit used for the developed autonomous drone system for power transmission line inspections weighs about $5.8 \mathrm{~kg}$. When using it in mountainous areas where vehicles cannot reach, staff must carry the drone, so it is necessary to consider the use of a smaller unit.

(b) Battery capacity

In the verification in this experiment at Location 2 (span length: $365 \mathrm{~m}$ ), it took 8 minutes and 40 seconds to inspect a power line when the drone was flown at $0.7 \mathrm{~m} / \mathrm{s}$. Considering the possible flight time of the drone, two power lines can be photographed in one flight, and then the batteries must be replaced. However, it is desirable for the drone to be able to capture the image of a three-phase circuit at a time in one flight. We have expectations for the future development of a battery with a larger capacity.

(c) Number of conductors to be inspected

The autonomous drone system for power transmission line inspections can be applied to flight along power lines and photography for the inspection of only single-conductor transmission lines. If an autonomous drone system for power transmission line inspections for multiple-conductor power lines is developed in the future, the applications will be drastically expanded.

(d) Automated power line fault diagnosis

When the obtained images are visually checked by humans, there is a limit to what we can do to improve efficiency. If the AI fault diagnosis developed in a previous study (10) becomes practical, the efficiency will be improved further. In order to introduce the AI fault diagnosis, the quality of images should be good enough that humans can find faults visually, and the images obtained in this study seem to satisfy this level of quality.

\section{Conclusions}

Using actual charged power transmission lines, we captured images of power lines with an autonomous drone system for power transmission line inspections, and confirmed that the system can be used for inspections of conductors and earth wires.

\section{References}

(1) Electric Technology Research Association: "Maintenance technology of overhead transmission facilities", Electric Technology Research, Vol. 76, No. 2, 2020 (in Japanese)

(2) Nobuhiro Kishigaki, Norihiko Tsutsumi, Kenshi Kanbara, Hiroyuki Nozaki, Tsuyoshi Chiba, Lopes Raphael, Takeshi Iida, Tsuyoshi Fujita: "Development of UAV Autonomous Flight Technique to Enable Parallel Flight along Dip of Overhead Power Transmission Line", Proc. 2018 Conf. Power \& Energy Society, IEE Japan, No. 302, pp. 9-5-17, 2018 (in Japanese) 
(3) Nobuhiro Kishigaki, Norihiko Tsutsumi, Kenshi Kanbara, Hiroyuki Nozaki, Tsuyoshi Chiba, Lopes Raphael, Kazuya Sakai, Masayuki Kumada, Takayuki Dewa, Tsuyoshi Fujita, Takeshi Iida: "Development of Autonomous Flight UAV with a Stereo Camera for acquiring electric wire image", 2019 IEEJ annual meeting, No. 7-128, pp. 208, 2019 (in Japanese)

(4) Takashi Yamamoto, Takatoshi Mimura, Yoichi Tomiki, Hirotaka Haji, Hiroyuki Nozaki: "Construction of Power Frequency Magnetic Field Immunity Test Facility for Drone", Proc. 2019 Conf. Power \& Energy Society, IEE Japan, No. 358, pp. 11-4-11, 2019 (in Japanese)

(5) Nobuhiro Kishigaki, Yoichi Tomiki, Takatoshi Mimura, Takashi Yamamoto, Norihiko Tsutsumi, Hiroyuki Nozaki, Hiroyuki Miyoshi, Kensei Yamamoto, Fumito Minoura, Tomoki Kitashima: “A Study on Separation Distance for Safe Navigation of Overhead Transmission Line Inspection Drone”, Proc. 2020 Conf. Power \& Energy Society, IEE Japan, No. 300, pp. 2WEB9-7, 2020 (in Japanese)

(6) Takatoshi Mimura, Takashi Yamamoto, Yoichi Tomiki, Nobuhiro Kishigaki, Hirotaka Haji, Hiroyuki Nozaki: "A Study on Minimum Approaching Distance of Drones to Power Lines", Proc. 2020 Conf. Power \& Energy Society, IEE Japan, No. 301, pp. 2WEB9-9, 2020 (in Japanese)

(7) Nobuhiro Kishigaki, Norihiko Tsutsumi, Kenshi Kanbara, Sakae Taniguchi, Hiroyuki Nozaki, Hiroyuki Miyoshi, Kensei Yamamoto, Fumito Minoura and Tomoki Kitashima, Tsuyoshi Chiba, Lopes Raphael, Tsuyoshi, Takayuki Dewa, Akihiko Ichihara: “Taking wire image in autonomous flight along overhead transmission line with sag”, 2020 IEEJ annual meeting, No. 7-107, pp. 173, 2020 (in Japanese)

(8) Masanobu Annoura, Toshiyuki Sakioka, Keisuke Sanda, Akira Fujii, Akira Ninohei, Kenichiro Fujita, Yosuke Tate: "The Development of Inspection Method for Ground Wire by Drone with Automatic Tracking Function”, Proc. 2019 Conf. Power \& Energy Society, IEE Japan, No. 357, pp. 11-4-9, 2019 (in Japanese)

(9) Hiroki Mizoe, Takahiro Kawamura, Etsuro Sato, Tatsuhiko Nakabayashi, Masaaki Maruyama: "Study on Overhead Transmission Line Inspection Using Drone - Automatic Tracking System for Wire Inspection Part 2 -", Proc. 2019 Conf. Power \& Energy Society, IEE Japan, No. 356, pp. 11-4-7, 2019 (in Japanese)
(10) Takuro Miyajima, Takahiro Uchimura, Shingo Esaka, Yoshio Sakamoto, Kazuki Iiduka, Aki Ito, Masaru Fujii, Tusuke Inoue, Noriaki Watanabe, Guanglomg Chi, Congying Zhang, Kohei shoji: "Development of Transmission line image diagnosis system utilized Artificial Intelligence", Proc. 2018 Conf. Power \& Energy Society, IEE Japan, No. 305, pp. 9-5-23, 2018 (in Japanese) 